\title{
Structure and VP16 binding of the Mediator Med25 activator interaction domain
}

\author{
Erika Vojnic ${ }^{1}$, André Mourão ${ }^{2-4}$, Martin Seizl $^{1}$, Bernd Simon $^{4}$, Larissa Wenzeck ${ }^{1}$, Laurent Larivière ${ }^{1}$, \\ Sonja Baumli ${ }^{1,6}$, Karen Baumgart ${ }^{5}$, Michael Meisterernst ${ }^{5}$, Michael Sattler ${ }^{2,3}$ \& Patrick Cramer $^{1}$
}

Eukaryotic transcription is regulated by interactions between gene-specific activators and the coactivator complex Mediator. Here we report the NMR structure of the Mediator subunit Med25 (also called Arc92) activator interaction domain (ACID) and analyze the structural and functional interaction of ACID with the archetypical acidic transcription activator VP16. Unlike other known activator targets, ACID forms a seven-stranded $\beta$-barrel framed by three helices. The VP16 subdomains $\mathrm{H} 1$ and $\mathrm{H} 2$ bind to opposite faces of ACID and cooperate during promoter-dependent activated transcription in a in vitro system. The activator-binding ACID faces are functionally required and conserved among higher eukaryotes. Comparison with published activator structures reveals that the VP16 activation domain uses distinct interaction modes to adapt to unrelated target surfaces and folds that evolved for activator binding.

Activator proteins regulate eukaryotic RNA polymerase II (Pol II) transcription in response to developmental and environmental signals. They bind to the DNA recognition sites of target genes with a sequence-specific DNA-binding domain, and recruit components of the Pol II machinery through a transcriptional activation domain $(\mathrm{TAD})^{1,2}$. Activators have been classified as acidic, glutamine-rich, proline-rich and serine/threonine-rich, depending on the preponderance of amino acids in their $\mathrm{TAD}^{3}$. An archetypal acidic activator is the herpes simplex virus protein 16 (VP16), which activates the expression of immediate early viral genes during infection ${ }^{4-8}$. VP16 exerts its activating function through a $\mathrm{C}$-terminal TAD that includes residues 413-490 (refs. 9-13).

The VP16 TAD has been intensely used for studying transcriptional activation. Usually, the VP16 TAD is fused with its $\mathrm{N}$ terminus to the DNA-binding domain of the yeast transcription factor Gal4. The resulting Gal4-VP16 activator fusion protein stimulates transcription from promoters that contain Gal4-binding sites in the yeast and mammalian transcription systems in vivo ${ }^{14,15}$ and in vitro ${ }^{16,17}$. This indicates that basic mechanisms of transcription activation are conserved amongst eukaryotes. The VP16 TAD targets basal Pol II transcription factors, including TFIIA, TFIIB, TFIID, the TFIIH subunit Tfb1/p62 (yeast/human) and the Mediator co-activator ${ }^{18-23}$. The VP16 TAD binds the Mediator subunit Med25 (also called Arc92) ${ }^{24-27}$, which is specific to higher eukaryotes. Med25 consists of two domains, the activator interaction domain (ACID) ${ }^{24}$ that binds the VP16 TAD, and a 'von Willebrand factor type A' domain that anchors Med25 to
Mediator ${ }^{24}$. Mediator generally conveys regulatory information by forming a bridge between activators and the basal Pol II machinery 28,29 . Whereas information on the core Mediator structure is emerging, structural information about its more peripheral activator-binding domains is limited to the KIX domain in subunit Med15 (or Arc105) 30,31

The VP16 TAD is intrinsically flexible, whereas the VP16 core domain (residues 49-402) forms a stable structure ${ }^{23,32-34}$. The TAD contains two functional subdomains, H1 (residues 410-452) and H2 (residues 453-490), which activate transcription independently ${ }^{35,36}$. Both $\mathrm{H} 1$ and $\mathrm{H} 2$ contain acidic amino acids, but specific bulky hydrophobic and aromatic residues are required for their function ${ }^{36-39}$. $\mathrm{H} 2$ has been proposed to adopt an $\alpha$-helical conformation when bound to $\mathrm{TFIIB}^{40}$. NMR analysis revealed a nine-residue amphipathic $\alpha$-helix in $\mathrm{H} 2$ that docks onto a $\mathrm{PH}$ fold in $\mathrm{Tfb}^{21}$. On the basis of these and other results it is assumed that acidic TADs are flexible in their free state, but become transiently structured upon interaction with their target proteins.

Here we report the NMR structure of the Mediator Med25 ACID and analyze its structural and functional interaction with the VP16 TAD. The structure revealed a fold that has not previously been described in activator target domains. NMR chemical shift perturbation analysis combined with biochemical assays showed that VP16 TAD interacts with an extended surface of Med25, in contrast to its known interaction with $\mathrm{Tfb} 1$. These results show that a canonical acidic TAD can adapt to unrelated target surfaces, and shows that diverse protein folds have evolved for TAD binding.

\footnotetext{
${ }^{1}$ Gene Center and Department of Biochemistry, Center for Integrated Protein Science Munich (CIPSM), Ludwig-Maximilians-Universität München, Munich, Germany. ${ }^{2}$ Institute of Structural Biology, Helmholtz Zentrum München, Neuherberg, Germany. ${ }^{3}$ Biomolecular NMR and Center for Integrated Protein Science Munich (CIPSM), Department Chemie, Garching, Germany. ${ }^{4}$ European Molecular Biology Laboratory (EMBL), Heidelberg, Germany. ${ }^{5}$ Institute of Molecular Tumor Biology, University of Muenster, Muenster, Germany. ${ }^{6}$ Present address: Laboratory of Molecular Biophysics, Department of Biochemistry, University of Oxford, Oxford, UK. Correspondence should be addressed to M.S. (sattler@helmholtz-muenchen.de) or P.C. (cramer@LMB.uni-muenchen.de).
} 
Figure 1 Solution structure of Med25 ACID. (a) Med25 domain architecture. (b) Stereo view of the backbone atoms ( $\mathrm{N}, \mathrm{C} \alpha$ and $\mathrm{C}^{\prime}$ ) of ten superimposed lowest-energy NMR structures. (c) Ribbon model for the lowest-energy conformer of ACID (left) and comparison with the SPOC domain of SHARP 42 (PDB 1OW1, right). Helices unique to SPOC are in green. Figures prepared with MOLMOL ${ }^{53}$ and PyMOL (http://www.pymol.org/).

\section{RESULTS}

\section{Med25 ACID solution structure}

We prepared recombinant human Med25 ACID (residues 394-543; Fig. 1a and Supplementary Fig. 1) and determined its solution structure using multidimensional NMR spectroscopy (Online Methods and Table 1). The secondary structure is defined by ${ }^{13} \mathrm{C}$ secondary chemical shifts and supported by H-D exchange experiments (Supplementary Fig. 2). The ensemble of ten lowest-energy conformers is shown in Figure 1b. ACID comprises a seven-stranded $\beta$-barrel framed by three $\alpha$-helices (Fig. 1c). The $\beta$-barrel consists of antiparallel strands ( $\beta 1$, Leu400-Trp408; $\beta 2$, Arg425-Asn434; $\beta 3$, Gln446-Pro454; $\beta 4$, Ser468-Phe475; $\beta 5$, Phe494-His499; $\beta 6$, Val510Ser516; $\beta 7$, Ile521-Ile526), with the exception of a short parallel arrangement between residues Val405 and Trp408 in $\beta 1$ and residues Lys447 and Met450 in $\beta 3$. Helix $\alpha 1$ (Gln455-Arg466), between $\beta 3$ and $\beta 4$, is slightly kinked at residues Gly462 and Pro463, resulting in a 3 -helix at residues Gly462-Arg466. Helix $\alpha 2$ (Leu480-Met490) connects strands $\beta 4$ and $\beta 5$, and helix $\alpha 3$ (Gln530-Asn543) forms the C-terminal region. Helix $\alpha 3$ is well defined by short-range NOEs. It is poised away from the central protein core, but nevertheless its orientation is well defined on the basis of eight NOEs to residues in the

\section{Table 1 NMR and refinement statistics for Med25 (Arc92) ACID}

NMR distance and dihedral constraints

Distance constraints ${ }^{a}$

Total NOE

1,597

Intra-residue

Inter-residue

$\begin{array}{lc}\text { Sequential }(|i-j|=1) & 499 \\ \text { Medium-range }(|i-j|<4) & 192 \\ \text { Long-range }(|i-j|>5) & 609 \\ \text { Hydrogen bonds } & 29\end{array}$

Total dihedral angle restraints ${ }^{b}$

$\phi$

117

$\psi$

123

\section{Structure statistics}

Violations (mean and s.d.)

Distance constraints $(\AA)$

Dihedral angle constraints $\left({ }^{\circ}\right)$

Max. dihedral angle violation $\left({ }^{\circ}\right)$

Max. distance constraint violation ( $\AA$ )

Deviations from idealized geometry

Bond lengths $(\AA)$

Bond angles $\left({ }^{\circ}\right)$

Average pairwise r.m.s. deviation $(\AA)$

Heavy

Backbone

\section{$0.307 \pm 0.082$ \\ $0.829 \pm 0.099$ \\ 5 \\ 0.5 \\ 0.017 \\ 1.4 \\ $1.22 \pm 0.10$ \\ $0.75 \pm 0.14$}

Statistics are given for the 10 lowest-energy structures after water refinement out of 100 calculated. We used the CNS $E_{\text {repel }}$ function to simulate van der Waals interactions with an energy constant of $25 \mathrm{kcal} \mathrm{mol}^{-1} \AA^{-4}$ using 'ROLSQ' van der Waals radii 56 . We calculated r.m.s. deviation using $\mathrm{MOLMOL}^{53}$. PROCHECK ${ }^{57}$ values apply for residues 395-409, 425-500 and 509-543.

aDistance restraints were employed with a soft square-well potential using an energy constant of $50 \mathrm{kcal} \mathrm{mol}^{-1} \AA^{-2}$. No distance restraint was violated by more than $0.5 \AA$. ' restraints derived from TALOS +58 were applied to $\varphi, \psi$ backbone angles using energy constants of $200 \mathrm{kcal} \mathrm{mol}^{-1} \mathrm{rad}^{-2}$.
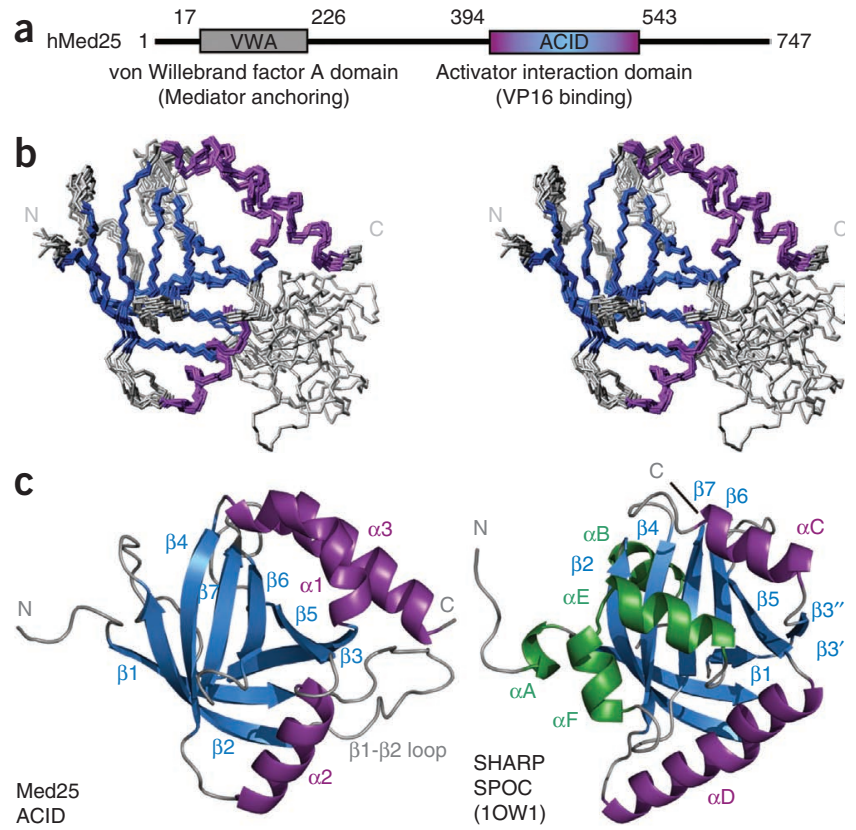

core domain. Loops $\beta 1-\beta 2$ (Lys411-Leu423), $\beta 2-\beta 3$ (His435-Lys440) and $\beta 5-\beta 6$ (Phe500-Arg509) contain highly mobile regions, consistent with the paucity of NOEs, ${ }^{13} \mathrm{C}$ secondary chemical shifts and low $\left\{{ }^{1} \mathrm{H}\right\}-{ }^{15} \mathrm{~N}$ heteronuclear NOE values (Supplementary Fig. 2).

\section{A novel activator target fold}

We carried out a structural similarity search with DALI ${ }^{41}$ but found no known activator target domains. However, the overall fold of Med25 ACID resembled the Spen paralog and ortholog C-terminal (SPOC) domain in the human transcriptional co-repressor SHARP ${ }^{42}$ (Fig. 1c) and the $\beta$-barrel fold in the Ku70 and Ku80 subunits of the heterodimeric DNA repair factor Ku70-Ku80 (ref. 43). The SPOC domain shares the central $\beta$-barrel and two flanking helices with Med25 ACID, but differs from the ACID structure by the presence of four additional helices (green in Fig. 1c). Helices $\alpha \mathrm{C}$ and $\alpha \mathrm{D}$ of the SPOC domain correspond topologically to helices $\alpha 1$ and $\alpha 2$, respectively, of ACID (Fig. 1c), whereas the SPOC domain also contains two $\mathrm{N}$-terminal and two $\mathrm{C}$-terminal helices. The N-terminal helices $\alpha \mathrm{A}$ and $\alpha \mathrm{B}$ enclose the first two strands in the barrel, whereas the $\mathrm{C}$-terminal helices $\alpha \mathrm{E}$ and $\alpha \mathrm{F}$ are inserted into a loop between $\beta 6$ and $\beta 7$. Compared to that of ACID, the central $\beta$-barrel of the SPOC domain shows a discontinuity in strand $\beta 3$ and a disruption of the characteristic pattern of hydrogen bonds between $\beta 3^{\prime}$ and the adjacent strand $\beta 5$. Therefore, ACID differs from the SPOC domain by the absence of four helices that flank the barrel in the SPOC domain and by the presence of a C-terminal helix $\alpha 3$ with a unique location and a long loop connecting $\beta 1$ and $\beta 2$.

\section{The VP16 TAD binds a large surface of Med25 ACID}

To study the binding of ACID to the VP16 TAD, we expressed the VP16 TAD as an N-terminal fusion protein with the Gal4 DNA-binding domain (see Supplementary Methods). Using a TEV protease cleavage site between the Gal4 domain and TAD, we prepared milligram quantities of a stable variant of VP16 TAD with a hexahistidine tag at its $\mathrm{C}$ terminus. To identify the surface of ACID that interacts with the VP16 TAD, we performed NMR chemical shift perturbation experiments. We recorded $2 \mathrm{D}{ }^{1} \mathrm{H},{ }^{15} \mathrm{~N}$ HSQC spectra of ${ }^{15} \mathrm{~N}$-labeled ACID during successive additions of VP16 TAD. Chemical shift 
Figure 2 VP16-ACID interaction. (a) Sequence of VP16 TAD with subdomains $\mathrm{H} 1$ and $\mathrm{H} 2$. Regions known to form a helical structure upon target interaction are underlined. Sites of mutation are highlighted with boxes (compare with Fig. 4c). (b) Overlay of the 2D ${ }^{1} \mathrm{H},{ }^{15} \mathrm{~N} \mathrm{HSQC}$ spectra for ${ }^{15} \mathrm{~N}$-labeled $\mathrm{ACID}$ in free form (black) and in the presence of 1.3 molar excess of VP16 TAD (red). Insets, chemical shift perturbations of specific residues upon addition of 0.2 (blue), 0.6 (purple), 1 (gold) and 1.3 (red) molar equivalents of TAD. (c) Histogram of the variation in chemical shift ( $\Delta \delta$ (p.p.m.)) observed in the 2D ${ }^{1} \mathrm{H},{ }^{15} \mathrm{~N}$ HSQC spectrum of ACID upon formation of the ACID-VP16 TAD complex at a molar ratio of $1: 1.3$. Red lines, residues with signals that were exchange-broadened, corresponding to red spheres in Figure $\mathbf{3}$ and Supplementary Figure $\mathbf{1}$. (d) Mapping of residues that undergo chemical shift changes ( $\Delta \delta>0.6$ p.p.m.) in the ${ }^{1} \mathrm{H},{ }^{15} \mathrm{~N}$ HSQC spectra upon binding of VP16 TAD to the ACID structure. Rising red color intensities correspond to increasing chemical shift changes. (e) Overlay of the $2 \mathrm{D}^{1} \mathrm{H},{ }^{15} \mathrm{~N}$ HSQC spectra for ${ }^{15} \mathrm{~N}$-labeled ACID in free form (black) and in the presence of an eight-fold molar excess of VP16 subdomain H2 (red). Insets, chemical shift perturbations of specific residues upon addition of 0.5 (blue), 1.5 (purple), 2 (gold) and 8 (red) molar equivalents of $\mathrm{H} 2$. (f) Histogram as in $\mathbf{c}$ but for VP16 $\mathrm{H} 2$ titration at a ratio of ACID: VP16 H2 of $1: 1.5$. (g) Mapping of residues that undergo chemical shift changes $(\Delta \delta>0.6$ p.p.m.) as in $\mathbf{d}$ but for VP16 $\mathrm{H} 2$ binding onto the ACID structure. a

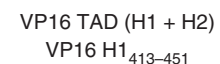

VP16 $\mathrm{H}_{413-451}$

西

Phe442 VP16 H2 $452-490 \quad$ Phe473 ${ }^{\text {Phe475 }}{ }_{\text {Phe479 }}$

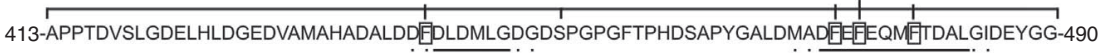
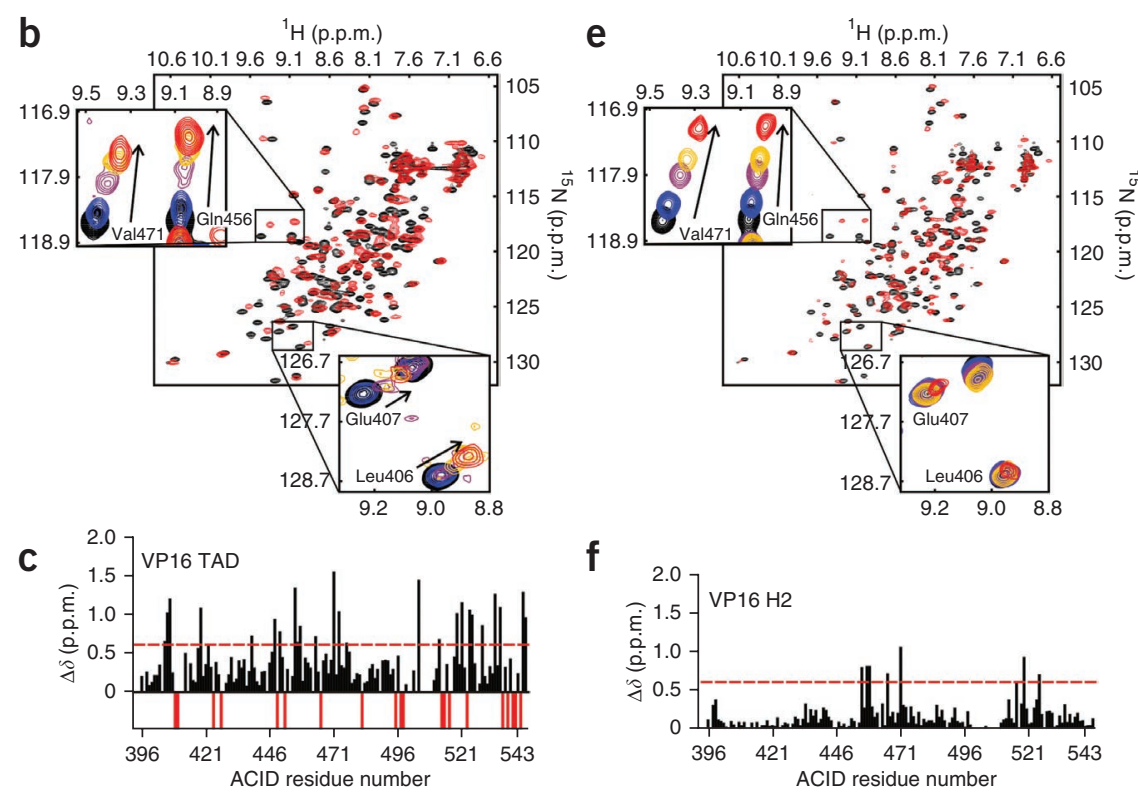

f
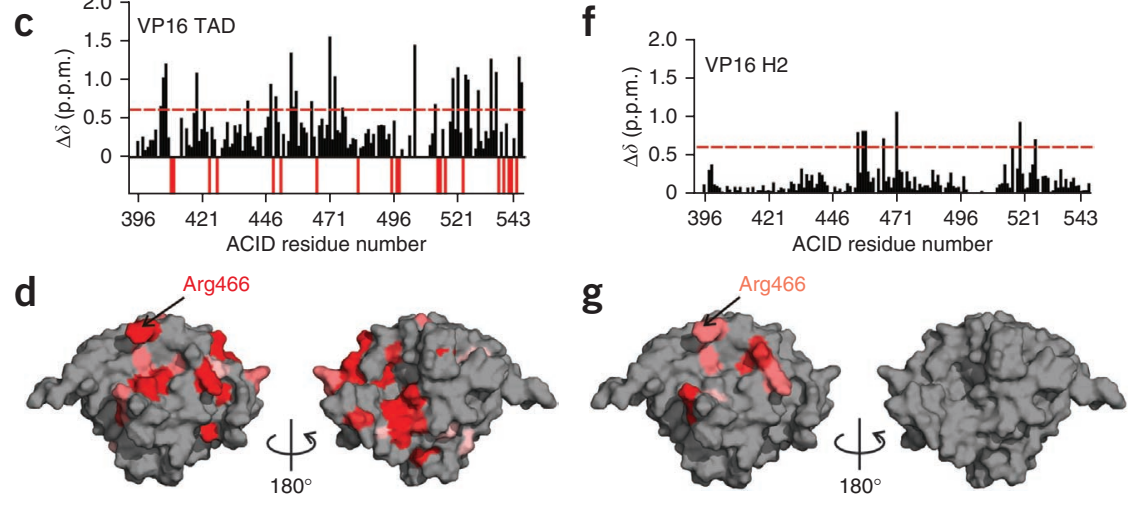

changes occurred upon TAD binding (Fig. 2a). The binding was in the fast to intermediate exchange regime on the NMR chemical shift time scale (Fig. 2). Saturation was achieved at about equimolar concentration of TAD and ACID, indicating a high-affinity interaction in the low micromolar to high nanomolar range. Numerous residues showed substantial chemical shift changes upon addition of VP16 TAD (Fig. 2 and Supplementary Fig. 1). Additional residues experienced substantial line-broadening, which suggests that there was conformational exchange in the binding interface (Figs. 2b-d, $\mathbf{3}$ and Supplementary Fig. 1). Mapping the affected residues onto the ACID structure revealed that VP16 TAD shared an extended binding interface with ACID (Fig. 2d). These data suggest that the VP16 TAD engages in

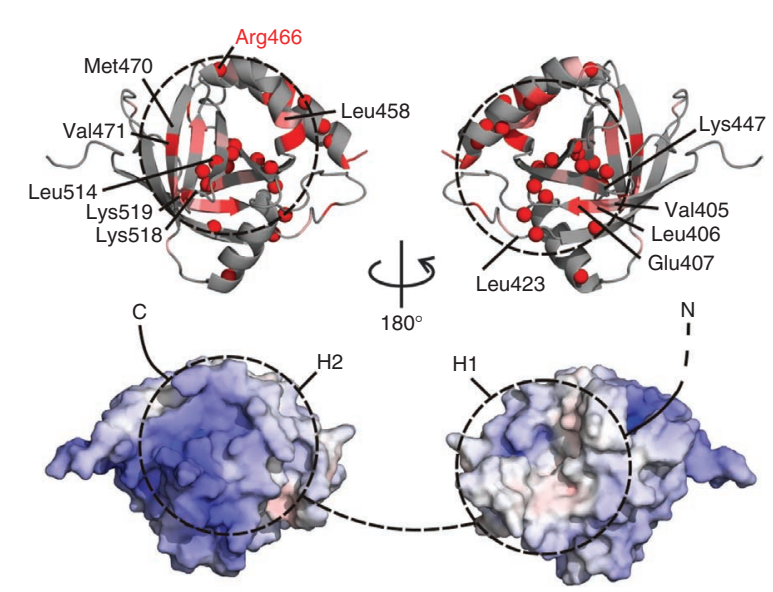

numerous contacts over a large surface area of ACID that comprises the central $\beta$-barrel and helices $\alpha 1$ and $\alpha 3$ at multiple sites.

\section{TAD subdomains $\mathrm{H} 1$ and $\mathrm{H} 2$ bind opposite ACID faces}

To investigate whether the two TAD subdomains contacted distinct patches on the extended ACID activator-binding surface, we carried out NMR titration experiments with the isolated $\mathrm{H} 2$ subdomain (Fig. 2e). Saturation was achieved only at eight-fold molar excess of $\mathrm{H} 2$ over ACID, indicating that the interaction with $\mathrm{H} 2$ was weaker than the interaction with the complete TAD. The NMR signals of many residues showed substantial line-broadening at two-fold molar excess of the VP16 TAD, presumably linked to conformational dynamics, and thus could not be further analyzed. However, there are already substantial chemical shift perturbations at a 1.5 -fold molar excess of $\mathrm{H} 2$ over ACID, affecting a number of residues on one face of the ACID structure (Fig. 2e-g). Residues on the opposite face, such as Leu 406 and Glu407, which were affected in the titration experiments with the complete TAD, did not show chemical shift changes upon addition of $\mathrm{H} 2$ even at saturating concentrations. More generally, residues that were perturbed by $\mathrm{H} 2$ binding formed a subset of the residues that were

Figure 3 VP16-binding interface of ACID. Two views of the ACID structure related by a $180^{\circ}$ rotation around a vertical axis are shown with residues perturbed upon VP16 binding (top) or as an electrostatic surface representation (bottom, blue and red for positive and negative charges, respectively). Rising red color intensities correspond to increasing chemical shift changes upon binding of VP16-TAD (Fig. 2b-d). Spheres indicate residues with signals that show binding in intermediate exchange (Fig. 2c and Supplementary Fig. 1). Dashed circles indicate opposite ACID faces interacting mainly with $\mathrm{H} 1$ and $\mathrm{H} 2$, respectively. 
Figure 4 Functional ACID-VP16 interaction. (a) EMSA supershift assay. The complex formed by DNA and Gal4-VP16 (lane 2) underwent a supershift with increasing concentrations of wild-type ACID (lanes 4-6). This supershift was abolished by ACID point mutation R466E (lane 7-9). (b) ACID quenched VP16 activation in a yeast transcription system. Assays were performed with yeast nuclear extracts (lane 1) or extracts with increasing amounts $(10,100$ or $400 \mathrm{pmol}$ ) of wild-type ACID (lanes 2-4) or ACID variant R466E (lanes 5-7). Transcription was quenched by recombinant ACID, but not by ACID variant R466E. (c) VP16 subdomains $\mathrm{H} 1$ and $\mathrm{H} 2$ activated yeast transcription in a synergistic, rather than competitive, manner. Transcription in yeast nuclear extracts was monitored in the absence (lanes 1, 10) or the presence of different Gal4-VP16 variants, including Gal4 fusions with VP16 TAD (lanes $2,3,11,12)$, TAD carrying the $\mathrm{H} 1$ subdomain mutation F442P (H1mt; lanes 4, 5), H1 (lanes 6, 7), H1mt (lanes 8, 9), TAD carrying the H2 subdomain mutations F473A F475A F479A (H2mt; lanes 13, 14), H2 (lanes 15, 16) and $\mathrm{H} 2 \mathrm{mt}$ (lanes 17, 18). (d) ACID inhibited transcription activation by VP16 in mammalian B-cell nuclear extracts. Assays were performed with nuclear extracts (lane 1 ) or extracts with increasing amounts (530 or 850 pmol) of wild-type ACID (lanes 2, 3) or specific ACID point mutants (lanes 4-13). The R466E variant of ACID hardly quenched transcription (lanes 4, 5), whereas other ACID variants did to various extents (lanes 6-13). Data are presented as average values of three experiments \pm s.d. and one representative gel is shown.

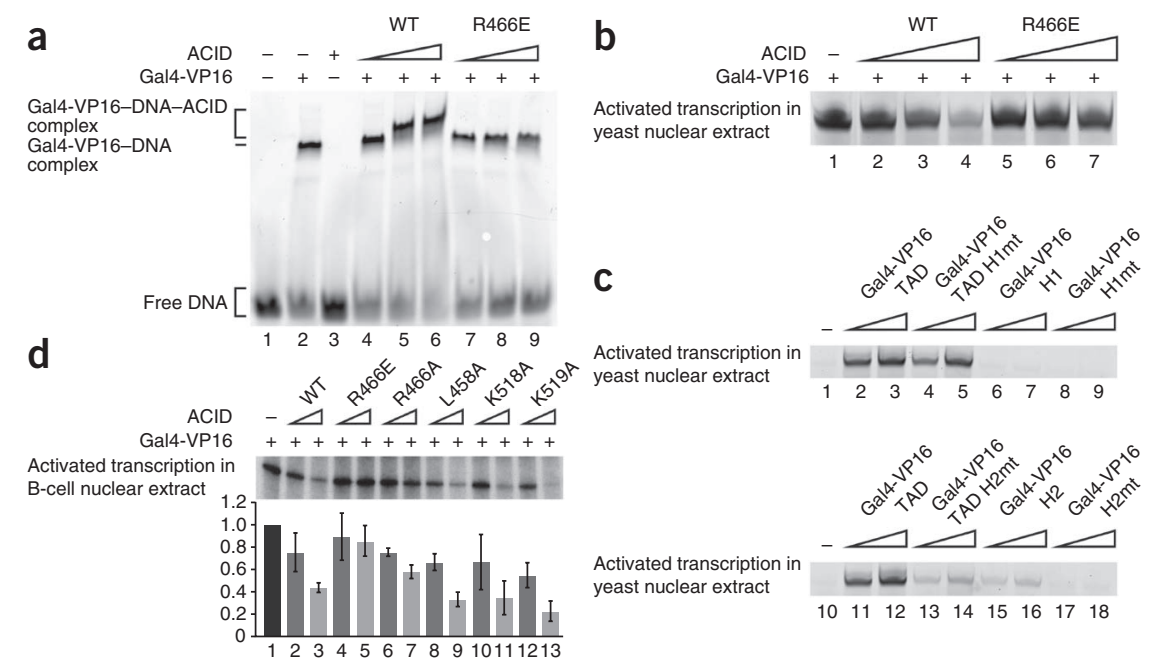

perturbed upon binding of the entire TAD. Whereas $\mathrm{H} 1$ binds to one ACID face, $\mathrm{H} 2$ binds to the opposite face of the domain (Fig. 3). Both subdomain-binding faces contain conserved exposed residues (Val405, Leu423 and Lys447 on the H1 face; Leu514, Arg466 and Met470 on the $\mathrm{H} 2$ face) that might interact with residues of VP16 required for its function ${ }^{37,39}$. These results indicate that the TAD subdomains H1 and $\mathrm{H} 2$ do not compete for the same ACID surface but rather bind to distinct $\mathrm{H} 1$ - and $\mathrm{H} 2$-binding patches on opposite faces of ACID.

\section{VP16-ACID interaction relies on a conserved basic residue}

To further investigate the TAD-ACID interaction biochemically, we used an electrophoretic mobility shift assay (EMSA; Online Methods). This assay used a fluorescently labeled DNA encompassing a Gal4binding site and allowed us to monitor the formation of a binary complex of the Gal4-VP16 fusion protein with DNA, and also the formation of a ternary complex of the Gal4-VP16-DNA complex with ACID (Fig. 4a). Formation of the ternary complex was seen as a supershift in the EMSA assay, and directly indicated an interaction between VP16 and ACID. To probe structural determinants in the interface, we mutated sites on ACID that showed strong perturbations in the NMR titration experiments. We generated 16 point mutant variants of ACID (Q455A, Q456A, L458A, T459A, T459V, R466E, R466A, S468A, V471A, V471L, L514A, S516A, K518A, K519A, L525V and L525A) and tested their interaction with VP16. All variants of ACID bound VP16 in the EMSA supershift assay (data not shown), except the charge-reversal variant R466E, which abolished the supershift (Fig. 4a). These results confirm that binding of VP16 to ACID is robust and might rely on multiple redundant contacts, and they show that residue Arg466 of ACID forms a crucial interaction 'hot spot'. This basic residue lies within the H2-binding face (Fig. 3) and is conserved among ACID homologs (Supplementary Fig. 1).

\section{Functional VP16 interaction with TAD-binding ACID surface}

To investigate whether the characterized interaction between VP16 and ACID is functionally important during activated transcription, we established a quenching assay. We performed in vitro activated transcription assays with yeast nuclear extracts on the HIS4 promoter with an upstream Gal4-binding site ${ }^{44}$. The Gal4-VP16 fusion protein is a potent transcriptional activator in this system (Fig. $\mathbf{4 b}$, lane 1). The addition of recombinant ACID quenches the transcription signal, apparently by competing with VP16 targets in the yeast extract (Fig. 4 b) lanes 2-4). Thus this assay monitors whether human ACID interferes with functional interactions between VP16 and the basal Pol II machinery during activated transcription in vitro. We tested five of the above recombinant ACID point mutant variants in the quenching assay. The variants quenched the transcription signal to different extents, but generally less than wild-type ACID, supporting the idea that the residues identified in NMR titration are involved in VP16 binding (Supplementary Fig. 3a, lanes 1-13). The ACID variant R466E had the strongest effect and only quenched transcription to a low extent (Fig. 4b, lanes 5-7), consistent with its key role in VP16 binding as observed in the EMSA assay.

\section{$\mathrm{H} 1$ and $\mathrm{H} 2$ cooperate during transcription activation in yeast} To evaluate the contributions of the VP16 subdomains to the activation of transcription in yeast, we prepared fusion proteins of the Gal4 DNAbinding domain with wild-type $\mathrm{H} 1$ or $\mathrm{H} 2$, with $\mathrm{H} 1$ or $\mathrm{H} 2$ mutated at functionally required hydrophobic residues, and with TAD mutated at

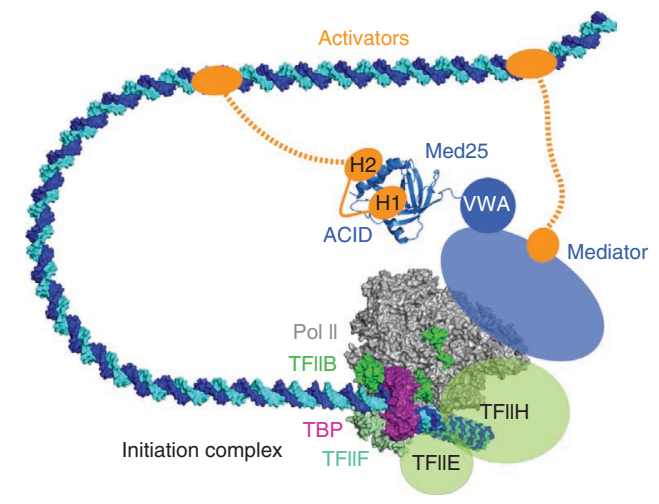

Figure 5 Model of an activated transcription initiation complex. A Pol II initiation complex was modeled on promoter DNA based on published results ${ }^{54,55}$, and DNA was extended with B-DNA. Blue, Mediator; orange, activators. 
functionally required hydrophobic residues in $\mathrm{H} 1$ or H2 (TAD H1mt and TAD H2mt, respectively; Fig. 2a). In these assays H2, but not H1, could activate transcription alone, albeit weakly (Fig. 4c, lanes 6, 7, 15, 16). Consistently, TAD that carried a mutation in $\mathrm{H} 2$ supported activated transcription only weakly (Fig. 4c, lanes $13,14)$, whereas TAD that carried a mutation in $\mathrm{H} 1$ strongly activated transcription, to nearly the levels of wild-type TAD (Fig. 4c, lanes 4, 5). Thus, the VP16 TAD subdomains $\mathrm{H} 1$ and $\mathrm{H} 2$ cooperate during activated transcription in the yeast system, with the main contribution coming from $\mathrm{H} 2$.

\section{The ACID-VP16 interaction is functional in the human system} Finally, we investigated whether the characterized VP16-ACID interaction is relevant in transcription activation in the human system. We investigated the quenching of activated transcription in human B-cell nuclear extracts by wild-type ACID and five ACID point mutant variants (Fig. 4d, lanes 1-13). Consistent with our observations in the yeast transcription system, the addition of wild-type ACID quenched transcriptional activation (Fig. 4d, lanes 2, 3) whereas addition of the R466E variant showed hardly any effect (Fig. 4d, lanes 4, 5). Furthermore, we studied the capability of ACID to interfere with another activator, SP1. ACID, but not the ACID variant R466E, quenched transcription activation by SP1 (Supplementary Fig. 3b, lanes 6-10), indicating that ACID uses the same binding surface for diverse activators. Thus, the VP16ACID interface characterized here by NMR is functionally relevant in the yeast and human transcription systems.

\section{DISCUSSION}

An important goal in the field of transcription regulation is to understand how activators function through the central coactivator complex Mediator (Fig. 5). Mediator integrates signals from activators bound upstream of the core promoter where Pol II and the basal factors assemble into the initiation complex. Structural information on activator-binding Mediator subunits and their activator interactions at a molecular level are an essential step toward this goal.

We have described the solution structure of the VP16 activator interaction domain in the human Mediator, the ACID domain in Mediator subunit Med25. We showed by NMR titration that VP16 binds to an extended surface of ACID and assigned two opposite faces to the interaction with the VP16 TAD subdomains $\mathrm{H} 1$ and $\mathrm{H} 2$. Consistently, $\mathrm{H} 1$ and $\mathrm{H} 2$ bind to ACID in a noncompetitive manner and cooperate during promoter-dependent activated transcription in yeast. The TAD-binding ACID surface is conserved among higher eukaryotes and is functionally relevant because it is required for efficient quenching of transcription in vitro.

Until now, structural information on activator-binding domains in

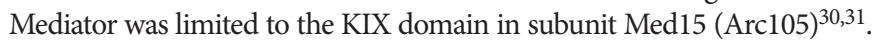
This domain shows an entirely $\alpha$-helical fold, in strong contrast to ACID, which contains a central $\beta$-barrel fold (Fig. 6). More generally, the fold of Med25 ACID is unique amongst known activator target domains ${ }^{21,45-47}$ such as Tfb1, Mdm2, PAS-B and the CBP KIX domain, which are all unrelated in structure (Fig. 6). However, there are two domains with a fold that resembles that of Med25 ACID in another human protein, PTOV1, which is overexpressed in prostate cancers ${ }^{48}$ and is involved in cell proliferation ${ }^{49}$ (Supplementary Fig. 4). As the amino acid residues of Med25 ACID that are involved in TAD binding are generally conserved in PTOV1, the two domains probably serve as activator-binding modules (Supplementary Fig. 4).

In contrast to the variety of target domain folds, activators form similar helical segments in all available structures of activator-target complexes ${ }^{21,45-47}$
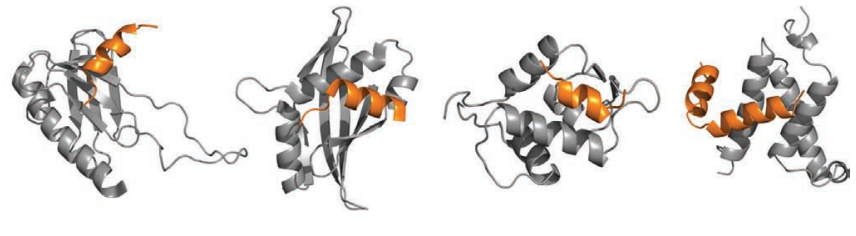

Tfb1-VP16 complex NcoA-1-STAT6 complex MDM2-p53 complex (2K2U) (10J5)

(1YCQ)

CBP-CREB complex (1KDX)

Figure 6 Comparison of ACID with known activator-target complexes. Activators are in orange and target domains are in silver. Depicted are ACID (this study), Tfb1-VP16 (ref. 21), NcoA-1-STAT6 (ref. 46), MDM2p53 (ref. 47) and CBP-KIX-CREB-pKID (ref. 45). PDB codes are in parentheses underneath each structure.

(Fig. 6). Unfortunately, we could not resolve an NMR structure of the VP16 TAD-ACID complex owing to substantial line-broadening of NMR signals in the binding interface (Supplementary Fig. 5). Nevertheless, analysis of ${ }^{13} \mathrm{C}$ secondary chemical shifts for the bound $\mathrm{H} 2$ peptide suggested that a central region comprising residues Ala471-Ala482 adopts a helical conformation (Supplementary Fig. 6). The line-broadening observed in both ACID and VP16 TAD suggests that the complex and the binding interface show conformational dynamics. As the ACID-TAD interaction is nevertheless tight and specific, this suggests that the activator might adopt multiple conformations on the same target surface. Such conformational flexibility is consistent with the general observation that activators form structured segments only transiently upon interaction with their targets through induced folding of helical segments ${ }^{23,50}$.

Conformation flexibility in TADs also explains the promiscuity of activators with respect to different target surfaces. In particular, VP16 binds different target proteins in the yeast and human systems. VP16 is a very strong activator in yeast even though yeast Mediator contains neither Med25 nor another subunit with ACID homology. Although the specific VP16 targets differ, VP16 TAD uses its two subdomains $\mathrm{H} 1$ and $\mathrm{H} 2$ in a synergistic manner in both systems, indicating that the yeast transcription machinery contains at least two nonoverlapping sites that can bind to the two TAD subdomains.

Our results provide insights into the evolution of Mediator and Mediatordependent transcription regulation. Whereas the yeast Mediator contains 25 subunits that are mostly conserved throughout the eukaryotes ${ }^{25}$, Mediator in higher eukaryotes contains additional, specific subunits and forms several distinct multiprotein subcomplexes ${ }^{51,52}$. The conserved Mediator core apparently contains a limited number of activator-binding target domains, whereas additional target domains are present on the extended surfaces of Mediator complexes from higher eukaryotes. The increase in target domain number and types might have made possible more complex transcriptional regulation. This might include processes that are specific to higher eukaryotes, such as differentiation or immune responses, and increased cooperativity might have allowed sharper on-off transitions. Surface extension of Mediator complexes during evolution was probably facilitated by the ability of activators to adapt to different targets.

\section{METHODS}

Methods and any associated references are available in the online version of the paper at http://www.nature.com/nsmb/.

Accession code. Coordinates of ten ACID structures have been deposited with the protein data bank under accession code $2 \mathrm{XNF}$.

Note: Supplementary information is available on the Nature Structural \& Molecular Biology website.

\section{ACKNOWLEDGMENTS}

We thank D. Kostrewa and K. Leike for help, and T. Fröhlich for help with MALDI and ESI experiments. M. Seizl was supported by the Boehringer 
Ingelheim Fonds and Elitenetzwerk Bayern. A.M. was supported by a Ph.D. fellowship (SFRH/BD/22323/2005) from the Portuguese Foundation for Science and Technology (FCT). M. Sattler acknowledges support by the Deutsche Forschungsgemeinschaft, the European Commission (3D Repertoire LSHGCT-2005-512028) and the Bavarian NMR Center. P.C. was supported by the Deutsche Forschungsgemeinschaft, the Sonderforschungsbereich SFB646, the SFB Transregio 5, the Jung-Stiftung and the Fonds der chemischen Industrie.

\section{AUTHOR CONTRIBUTIONS}

E.V., A.M. and B.S., NMR data acquisition and analysis; E.V., M. Seizl, L.W., L.L. and S.B., sample preparation and functional assays; K.B. and M.M., mammalian transcription assays; M.M., M. Sattler and P.C., project design and supervision; E.V., M. Sattler and P.C., manuscript preparation.

\section{COMPETING FINANCIAL INTERESTS}

The authors declare no competing financial interests.

Published online at http://www.nature.com/nsmb/.

Reprints and permissions information is available online at http://npg.nature.com/ reprintsandpermissions/.

1. Brent, R. \& Ptashne, M. A eukaryotic transcriptional activator bearing the DNA specificity of a prokaryotic repressor. Cell 43, 729-736 (1985).

2. Keegan, L., Gill, G. \& Ptashne, M. Separation of DNA binding from the transcriptionactivating function of a eukaryotic regulatory protein. Science 231, 699-704 (1986).

3. Mitchell, P.J. \& Tjian, R. Transcriptional regulation in mammalian cells by sequencespecific DNA binding proteins. Science 245, 371-378 (1989).

4. Gerster, T. \& Roeder, R.G. A herpesvirus trans-activating protein interacts with transcription factor OTF-1 and other cellular proteins. Proc. Natl. Acad. Sci. USA 85, 6347-6351 (1988).

5. Katan, M., Haigh, A., Verrijzer, C.P., van der Vliet, P.C. \& O'Hare, P. Characterization of a cellular factor which interacts functionally with Oct-1 in the assembly of a multicomponent transcription complex. Nucleic Acids Res. 18, 6871-6880 (1990).

6. Stern, S., Tanaka, M. \& Herr, W. The Oct-1 homoeodomain directs formation of a multiprotein-DNA complex with the HSV transactivator VP16. Nature 341, 624-630 (1989).

7. Wilson, A.C., LaMarco, K., Peterson, M.G. \& Herr, W. The VP16 accessory protein HCF is a family of polypeptides processed from a large precursor protein. Cell 74 , 115-125 (1993)

8. Wysocka, J. \& Herr, W. The herpes simplex virus VP16-induced complex: the makings of a regulatory switch. Trends Biochem. Sci. 28, 294-304 (2003).

9. Greaves, R. \& O'Hare, P. Separation of requirements for protein-DNA complex assembly from those for functional activity in the herpes simplex virus regulatory protein Vmw65. J. Virol. 63, 1641-1650 (1989).

10. Greaves, R.F. \& O'Hare, P. Structural requirements in the herpes simplex virus type 1 transactivator Vmw65 for interaction with the cellular octamer-binding protein and target TAATGARAT sequences. J. Virol. 64, 2716-2724 (1990).

11. Lai, J.S. \& Herr, W. Interdigitated residues within a small region of VP16 interact with Oct-1, HCF, and DNA. Mol. Cell. Biol. 17, 3937-3946 (1997).

12. Triezenberg, S.J., Kingsbury, R.C. \& McKnight, S.L. Functional dissection of VP16, the trans-activator of herpes simplex virus immediate early gene expression. Genes Dev. 2, 718-729 (1988a).

13. Triezenberg, S.J., LaMarco, K.L. \& McKnight, S.L. Evidence of DNA: protein interactions that mediate HSV-1 immediate early gene activation by VP16. Genes Dev. 2, 730-742 (1988b).

14. Sadowski, I., Ma, J., Triezenberg, S. \& Ptashne, M. GAL4-VP16 is an unusually potent transcriptional activator. Nature 335, 563-564 (1988).

15. Cousens, D.J., Greaves, R., Goding, C.R. \& O'Hare, P. The C-terminal 79 amino acids of the herpes simplex virus regulatory protein, $V m w 65$, efficiently activate transcription in yeast and mammalian cells in chimeric DNA-binding proteins. EMBO J. 8, 2337-2342 (1989).

16. Berger, S.L., Cress, W.D., Cress, A., Triezenberg, S.J. \& Guarente, L. Selective inhibition of activated but not basal transcription by the acidic activation domain of VP16: evidence for transcriptional adaptors. Cel/ 61, 1199-1208 (1990).

17. Chasman, D.I., Leatherwood, J., Carey, M., Ptashne, M. \& Kornberg, R.D. Activation of yeast polymerase II transcription by herpesvirus VP16 and GAL4 derivatives in vitro. Mol. Cell. Biol. 9, 4746-4749 (1989).

18. Kobayashi, N., Boyer, T.G. \& Berk, A.J. A class of activation domains interacts directly with TFIIA and stimulates TFIIA-TFIID-promoter complex assembly. Mol. Cell. Biol. 15, 6465-6473 (1995).

19. Kobayashi, N. et al. DA-complex assembly activity required for VP16C transcriptional activation. Mol. Cell. Biol. 18, 4023-4031 (1998).

20. Hall, D.B. \& Struhl, K. The VP16 activation domain interacts with multiple transcriptional components as determined by protein-protein cross-linking in vivo. J. Biol. Chem. 277, 46043-46050 (2002).

21. Langlois, C. et al. NMR structure of the complex between the Tfb1 subunit of TFIIH and the activation domain of VP16: structural similarities between VP16 and p53. J. Am. Chem. Soc. 130, 10596-10604 (2008).

22. Xiao, $\mathrm{H}$. et al. Binding of basal transcription factor TFIIH to the acidic activation domains of VP16 and p53. Mol. Cell. Biol. 14, 7013-7024 (1994).

23. Uesugi, M., Nyanguile, O., Lu, H., Levine, A.J. \& Verdine, G.L. Induced alpha helix in the VP16 activation domain upon binding to a human TAF. Science 277, 1310-1313 (1997).
24. Mittler, G. et al. A novel docking site on Mediator is critical for activation by VP16 in mammalian cells. EMBO J. 22, 6494-6504 (2003).

25. Bourbon, H.M. et al. A unified nomenclature for protein subunits of mediator complexes linking transcriptional regulators to RNA polymerase II. Mol. Cell 14, 553-557 (2004).

26. Näär, A.M. et al. Composite co-activator ARC mediates chromatin-directed transcriptional activation. Nature 398, 828-832 (1999).

27. Yang, F., DeBeaumont, R., Zhou, S. \& Naar, A.M. The activator-recruited cofactor/ Mediator coactivator subunit ARC92 is a functionally important target of the VP16 transcriptional activator. Proc. Natl. Acad. Sci. USA 101, 2339-2344 (2004).

28. Kornberg, R.D. Mediator and the mechanism of transcriptional activation. Trends Biochem. Sci. 30, 235-239 (2005).

29. Malik, S. \& Roeder, R.G. Dynamic regulation of pol II transcription by the mammalian Mediator complex. Trends Biochem. Sci. 30, 256-263 (2005).

30. Thakur, J.K. et al. A nuclear receptor-like pathway regulating multidrug resistance in fungi. Nature 452, 604-609 (2008).

31. Yang, F. et al. An ARC/Mediator subunit required for SREBP control of cholesterol and lipid homeostasis. Nature 442, 700-704 (2006).

32. Grossmann, J.G., Sharff, A.J., O'Hare, P. \& Luisi, B. Molecular shapes of transcription factors TFIIB and VP16 in solution: implications for recognition. Biochemistry $\mathbf{4 0}$, 6267-6274 (2001)

33. Kim, D.H. et al. Multiple hTAF(II)31-binding motifs in the intrinsically unfolded transcriptional activation domain of VP16. BMB Rep. 42, 411-417 (2009).

34. Liu, Y., Gong, W., Huang, C.C., Herr, W. \& Cheng, X. Crystal structure of the conserved core of the herpes simplex virus transcriptional regulatory protein VP16. Genes Dev. 13, 1692-1703 (1999).

35. Ikeda, K., Stuehler, T. \& Meisterernst, M. The $\mathrm{H} 1$ and $\mathrm{H} 2$ regions of the activation domain of herpes simplex virion protein 16 stimulate transcription through distinct molecular mechanisms. Genes Cells 7, 49-58 (2002).

36. Walker, S., Greaves, R. \& O'Hare, P. Transcriptional activation by the acidic domain of Vmw65 requires the integrity of the domain and involves additional determinants distinct from those necessary for TFIIB binding. Mol. Cell. Biol. 13, 5233-5244 (1993).

37. Cress, W.D. \& Triezenberg, S.J. Critical structural elements of the VP16 transcriptional activation domain. Science 251, 87-90 (1991)

38. Regier, J.L., Shen, F. \& Triezenberg, S.J. Pattern of aromatic and hydrophobic amino acids critical for one of two subdomains of the VP16 transcriptional activator. Proc. Natl. Acad. Sci. USA 90, 883-887 (1993).

39. Sullivan, S.M. et al. Mutational analysis of a transcriptional activation region of the VP16 protein of herpes simplex virus. Nucleic Acids Res. 26, 4487-4496 (1998).

40. Jonker, H.R., Wechselberger, R.W., Boelens, R., Folkers, G.E. \& Kaptein, R. Structural properties of the promiscuous VP16 activation domain. Biochemistry 44 827-839 (2005).

41. Holm, L. \& Sander, C. Dali: a network tool for protein structure comparison. Trends Biochem. Sci. 20, 478-480 (1995).

42. Ariyoshi, M. \& Schwabe, J.W. A conserved structural motif reveals the essential transcriptional repression function of Spen proteins and their role in developmental signaling. Genes Dev. 17, 1909-1920 (2003).

43. Walker, J.R., Corpina, R.A. \& Goldberg, J. Structure of the Ku heterodimer bound to DNA and its implications for double-strand break repair. Nature 412, 607-614 (2001).

44. Ranish, J.A. \& Hahn, S. The yeast general transcription factor TFIIA is composed of two polypeptide subunits. J. Biol. Chem. 266, 19320-19327 (1991).

45. Radhakrishnan, I. et al. Solution structure of the KIX domain of CBP bound to the transactivation domain of CREB: a model for activator:coactivator interactions. Cell 91, 741-752 (1997).

46. Razeto, A. et al. Structure of the NCoA-1/SRC-1 PAS-B domain bound to the LXXLL motif of the STAT6 transactivation domain. J. Mol. Biol. 336, 319-329 (2004).

47. Kussie, P.H. et al. Structure of the MDM2 oncoprotein bound to the p53 tumor suppressor transactivation domain. Science $\mathbf{2 7 4}$, 948-953 (1996).

48. Benedit, P. et al. PTOV1, a novel protein overexpressed in prostate cancer containing a new class of protein homology blocks. Oncogene 20, 1455-1464 (2001).

49. Santamaría, A. et al. PTOV1 enables the nuclear translocation and mitogenic activity of flotillin-1, a major protein of lipid rafts. Mol. Cell. Biol. 25, 1900-1911 (2005).

50. McEwan, I.J., Dahlman-Wright, K., Ford, J. \& Wright, A.P. Functional interaction of the c-Myc transactivation domain with the TATA binding protein: evidence for an induced fit model of transactivation domain folding. Biochemistry 35, 9584-9593 (1996).

51. D'Alessio, J.A., Wright, K.J. \& Tjian, R. Shifting players and paradigms in cellspecific transcription. Mol. Cell 36, 924-931 (2009).

52. Levine, M. \& Tjian, R. Transcription regulation and animal diversity. Nature $\mathbf{4 2 4}$ 147-151 (2003)

53. Koradi, R., Billeter, M. \& Wuthrich, K. MOLMOL: a program for display and analysis of macromolecular structures. J. Mol. Graph. 14, 51-55 (1996).

54. Chen, Z.A. et al. Architecture of the RNA polymerase II-TFIIF complex revealed by cross-linking and mass spectrometry. EMBO J. 29, 717-726 (2010).

55. Kostrewa, D. et al. RNA polymerase II-TFIIB structure and mechanism of transcription initiation. Nature 462, 323-330 (2009).

56. Linge, J.P., Williams, M.A., Spronk, C.A., Bonvin, A.M. \& Nilges, M. Refinement of protein structures in explicit solvent. Proteins 50, 496-506 (2003)

57. Laskowski, R.A., Rullmannn, J.A., MacArthur, M.W., Kaptein, R. \& Thornton, J.M. AQUA and PROCHECK-NMR: programs for checking the quality of protein structures solved by NMR. J. Biomol. NMR 8, 477-486 (1996).

58. Shen, Y., Delaglio, F., Cornilescu, G. \& Bax, A. TALOS+: a hybrid method for predicting protein backbone torsion angles from NMR chemical shifts. J. Biomol. NMR 44, 213-223 (2009). 


\section{ONLINE METHODS}

Plasmids and proteins. Details of cloning, protein expression and purification are described in Supplementary Methods.

NMR spectroscopy. All NMR spectra were recorded at $295 \mathrm{~K}$ on Bruker DRX600 and 900 spectrometers with cryogenic triple resonance probes using a ${ }^{13} \mathrm{C},{ }^{15} \mathrm{~N}$ labeled ACID sample. Data were processed with NMRPipe ${ }^{59}$ and analyzed using $\mathrm{NMRView}^{60}$. Backbone sequential resonances were assigned using 3D HNCA, $\mathrm{HNCACB}, \mathrm{HN}(\mathrm{CO}) \mathrm{CA}, \mathrm{HN}(\mathrm{CO}) \mathrm{CACB}$ experiments and side chain resonances were assigned using 3D HCCH-TOCSY experiments ${ }^{61}$. Secondary structure elements were identified by analysis of ${ }^{13} \mathrm{C}$ secondary chemical shifts and $\mathrm{H}-\mathrm{D}$ exchange experiments as well as NOE pattern. Distance constraints were collected from $3 \mathrm{D}{ }^{15} \mathrm{~N}$-edited NOESY $\left(\tau_{\mathrm{m}}=90 \mathrm{~ms}\right), 3 \mathrm{D}{ }^{13} \mathrm{C}$-edited NOESY $\left(\tau_{\mathrm{m}}=90 \mathrm{~ms}\right)$ and $2 \mathrm{D}^{1} \mathrm{H}$-NOESY $\left(\tau_{\mathrm{m}}=90 \mathrm{~ms}\right)$ spectra ${ }^{61}$. Information on backbone dynamics was derived from ${ }^{15} \mathrm{~N}$ relaxation experiments comprising measurements of $\mathrm{T} 1$, T2 and $\left\{{ }^{1} \mathrm{H}-\right\}^{15} \mathrm{~N}$ heteronuclear NOEs ${ }^{62}$.

The interactions of VP16 H2 and VP16 TAD with Med25 ACID were monitored by comparing $2 \mathrm{D}{ }^{1} \mathrm{H},{ }^{15} \mathrm{~N}$ HSQC spectra of the ${ }^{13} \mathrm{C},{ }^{15} \mathrm{~N}$-labeled VP16 H2 $(0.8 \mathrm{mM})$ or VP16 TAD $(0.28 \mathrm{mM})$ protein alone and in the presence of equimolar unlabeled ACID protein at $295 \mathrm{~K}$ in $2.5 \mathrm{mM}$ sodium phosphate, $\mathrm{pH} 6.5,100$ $\mathrm{mM} \mathrm{NaCl}, 4 \mathrm{mM}$ DTT and $10 \%$ (v/v) $\mathrm{D}_{2} \mathrm{O}$ on Bruker $600-\mathrm{MHz}(\mathrm{VP} 16 \mathrm{H} 2)$ or 900-MHz (VP16 TAD) spectrometers equipped with a cryoprobe. For ${ }^{13} \mathrm{C},{ }^{15} \mathrm{~N}$ labeled VP16 H2, backbone experiments were recorded.

Structure calculations. Automated NOE assignment and structure calculations were carried out with the standard protocol in CYANA version 2.1 (ref. 63). After seven iterative cycles of automated NOE assignment, $85 \%$ of NOESY peaks were assigned. NOEs assignments and completeness of NOEs were manually checked. NOE-derived distance restraints from CYANA calculations, dihedral angle restraints derived from TALOS $+{ }^{58}$, and hydrogen bonds derived from $\mathrm{H}-\mathrm{D}$ exchange experiments were applied in a simulated annealing protocol in $\mathrm{CNS}^{64}$ for a water-refinement calculation ${ }^{56}$. Out of 100 calculated structures, an ensemble of ten structures with the lowest energy was used for validation with PROCHECK-NMR ${ }^{57}$ and iCING (G.W. Vuister, Univ. Nijmegen; http:// nmr.cmbi.ru.nl/icing/). Ramachandran plot analysis of the final structures, including residues 395-409, 425-500 and 509-543, with PROCHECK-NMR ${ }^{57}$ showed that $92.0 \%, 7.1 \%, 0.5 \%$ and $0.4 \%$ of residues are in the most favored, additionally allowed, generously allowed and disallowed regions, respectively. WHATCHECK ${ }^{65}$ was used for analysis using the iCING web server.

NMR titration. Chemical shift mapping on ACID was done by monitoring the 2D ${ }^{1} \mathrm{H},{ }^{15} \mathrm{~N}$ HSQC spectra of the uniformly ${ }^{15} \mathrm{~N}$-labeled ACID protein alone $(0.22-0.3 \mathrm{mM})$ and with an excess of unlabeled interacting proteins until no further changes in chemical shifts were observed in the $2 \mathrm{D}{ }^{1} \mathrm{H},{ }^{15} \mathrm{~N}$ HSQC spectra. The HSQC spectra were recorded at $295 \mathrm{~K}$ in $2.5 \mathrm{mM}$ sodium phosphate, pH 6.5, $100 \mathrm{mM} \mathrm{NaCl}, 4 \mathrm{mM}$ DTT and 10\% (v/v) D $2 \mathrm{O}$ on a Bruker $600 \mathrm{MHz}$ spectrometer equipped with a cryoprobe. The values were calculated using the equation $\Delta \delta=\left\{\left[\Delta \delta\left({ }^{1} \mathrm{H}\right)^{2}+\left[0.2^{\star} \Delta \delta\left({ }^{15} \mathrm{~N}\right)\right]\right\}^{1 / 2}\right.$.

Electrophoretic mobility shift assay. Binding reactions $(20 \mu \mathrm{l})$ contained $5 \mathrm{pmol}$ Gal4-VP16 and 5, 50 or 150 pmol ACID variants. We used 1 pmol of a DNA duplex with sequence $5^{\prime}$-GGGGATCGATCCGGGTGACAGCCCTCCGAATTC GAGCTCG-3', containing a single Gal4-binding site (underlined). After incubation for $20 \mathrm{~min}$ at room temperature, ACID variants were added accordingly and the mixture was incubated for $10 \mathrm{~min}$. Formed complexes were separated on $5 \%$ (v/v) acrylamide gels in TGOE buffer (0.25 M Tris, $\mathrm{pH} 8.3,1.9 \mathrm{M}$ glycine). Bands were quantified with a Typhoon 9400 scanner and the ImageQuant Software (Amersham Biosciences).

Activated transcription in nuclear extracts. Yeast nuclear extracts were prepared from three liters of culture as described (http://www.fhcrc.org/labs/hahn). Yeast in vitro transcription and analysis by primer extension were as described ${ }^{66}$. Reactions contained $150 \mu \mathrm{g}$ nuclear extracts, 5 pmol Gal4-VP16 and 10, 100 or 400 pmol ACID variant. All in vitro transcription reactions with the mammalian B-cell nuclear extract were performed in a $20 \mu \mathrm{l}$ reaction volume. The standard reaction mixture contained $25 \mathrm{mM}$ HEPES, pH 8.2, $4 \mathrm{mM} \mathrm{MgCl}_{2}, 2.5 \mathrm{mM}$ DTT, 20 U RiboLock RNase Inhibitor, $0.5 \mathrm{mg} \mathrm{ml}^{-1} \mathrm{BSA}, 0.005 \%$ (v/v) Igepal, $2.5 \mu \mathrm{M}$ ATP, $1 \times$ Protease inhibitor (EDTA free), 100 ng pGalML linearized with SmaI, $6.5 \mu \mathrm{l}$ Raji cell nuclear extract, 530 or 850 pmol ACID variant and 1.6 pmol Gal4VP16 or 3.5 pmol Gal94-SP1. HEPES, $\mathrm{MgCl}_{2}$, DTT, RiboLock RNase Inhibitor, BSA, Igepal, ATP, Protease inhibitor and linearized pGalML were mixed with the ACID variant, ACID buffer and Gal4-VP16 or Gal94-SP1. Following $12 \mathrm{~min}$ incubation at $25^{\circ} \mathrm{C}$ the nuclear extract was added and the reaction mixture was centrifuged and incubated for $30 \mathrm{~min}$ at $25^{\circ} \mathrm{C}$. Afterwards $5 \mu \mathrm{Ci}\left(\alpha_{-}{ }^{32} \mathrm{P}\right) \mathrm{UTP}$ (3000 $\mathrm{Ci} \mathrm{mmol}^{-1}$ ), 0,1 $\mu \mathrm{M}$ ATP, CTP, GTP and $10 \mu \mathrm{M}$ UTP were added and the reaction mixture incubated for $1 \mathrm{~h}$ at $25^{\circ} \mathrm{C}$. Subsequently $25 \mathrm{mM}$ GTP, ATP, CTP, and UTP were added, the mixture was centrifuged and the reaction was continued for $15 \mathrm{~min}$ at $30{ }^{\circ} \mathrm{C}$. In vitro transcription reactions were stopped by adding $200 \mu \mathrm{g}$ yeast tRNA and $400 \mu \mathrm{l}$ transcription stop buffer ( $7 \mathrm{M}$ urea, 10 $\mathrm{mM}$ Tris- $\mathrm{HCl}, \mathrm{pH}$ 7.8, 10 mM EDTA/NaOH, pH 8.0, 0.5\% (v/v) SDS, $100 \mathrm{mM}$ $\mathrm{LiCl}, 300 \mathrm{mM}$, sodium acetate, $\mathrm{pH}$ 5.2) and the RNA was isolated by phenolchloroform extraction, precipitated with isopropanol at $-20^{\circ} \mathrm{C}$ for at least $2 \mathrm{~h}$, pelleted by centrifugation $\left(45 \mathrm{~min}, 4^{\circ} \mathrm{C}, 16,873 \mathrm{~g}\right.$ ) washed in each case once with $70 \%(\mathrm{v} / \mathrm{v})$ and $100 \%$ ethanol, air dried for $15 \mathrm{~min}$ and then resuspended in $10 \mu \mathrm{l}$ formamid loading buffer. RNA samples were shaken for $15 \mathrm{~min}$ at $50^{\circ} \mathrm{C}$ and afterwards loaded on a $5 \%(\mathrm{v} / \mathrm{v})$ polyacrylamide gel with $8 \mathrm{M}$ urea. The gel was run at constant current $(50 \mathrm{~mA})$ for $1 \mathrm{~h}$ and $50 \mathrm{~min}$, and dried for one hour at $80^{\circ} \mathrm{C}$ on the vacuum gel dryer. The dried gel was exposed to either a KODAK BioMax MS film (Scientific Imaging Film) or a phosphor-imager plate. Quantification was performed with the Personal Molecular Imager FX (Bio-Rad) and Quantity One 4.6.9. software.

59. Delaglio, F. et al. NMRPipe: a multidimensional spectral processing system based on UNIX pipes. J. Biomol. NMR 6, 277-293 (1995).

60. Johnson, B.A. \& Blevins, R.A. NMRView: a computer program for the visualization and analysis of NMR data. J. Biomol. NMR 4, 603-614 (1994).

61. Sattler, M., Schleucher, J. \& Griesinger, C. Heteronuclear multidimensional NMR experiments for the structure determination of proteins in solution employing pulsed field gradients. Prog. Nucl. Magn. Reson. Spectrosc. 34, 93-158 (1999).

62. Farrow, N.A. et al. Backbone dynamics of a free and phosphopeptide-complexed Src homology 2 domain studied by $15 \mathrm{~N}$ NMR relaxation. Biochemistry 33, 5984-6003 (1994).

63. Güntert, P. Automated NMR structure calculation with CYANA. Methods Mol. Biol. 278, 353-378 (2004).

64. Brünger, A.T. et al. Crystallography \& NMR system: A new software suite for macromolecular structure determination. Acta Crystallogr. D Biol. Crystallogr. 54, 905-921 (1998).

65. Hooft, R.W., Vriend, G., Sander, C. \& Abola, E.E. Errors in protein structures. Nature 381, 272 (1996).

66. Larivière, L. et al. Structure-system correlation identifies a gene regulatory Mediator submodule. Genes Dev. 22, 872-877 (2008). 